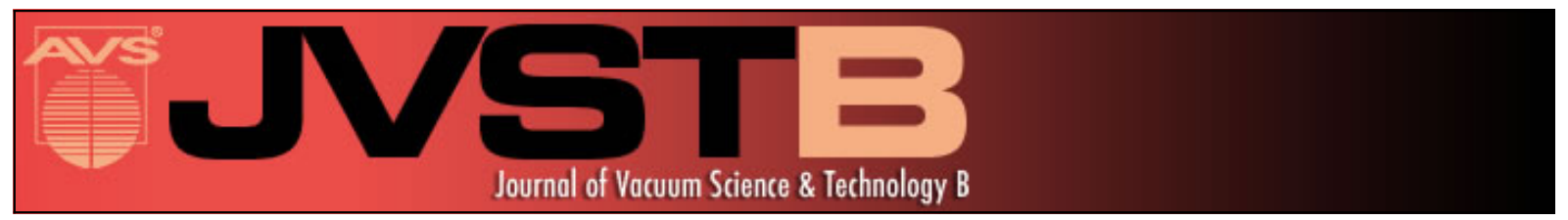

\title{
Room-temperature nanoimprinting on metallo-organic complexes
}

Elisa Mele, Dario Pisignano, Marco Mazzeo, Luana Persano, Giuseppe Gigli, and Roberto Cingolani

Citation: Journal of Vacuum Science \& Technology B 22, 981 (2004); doi: 10.1116/1.1714952

View online: http://dx.doi.org/10.1116/1.1714952

View Table of Contents: http://scitation.aip.org/content/avs/journal/jvstb/22/3?ver=pdfcov

Published by the AVS: Science \& Technology of Materials, Interfaces, and Processing

\section{Articles you may be interested in}

Low-voltage organic field-effect transistors based on novel high-k organometallic lanthanide complex for gate insulating materials

AlP Advances 4, 087140 (2014); 10.1063/1.4894450

Role of spatial distortions on the quadratic nonlinear optical properties of octupolar organic and metallo-organic molecules

J. Chem. Phys. 126, 034312 (2007); 10.1063/1.2428308

Color tunable organic light-emitting diodes by using europium organometallic complex

Appl. Phys. Lett. 89, 251108 (2006); 10.1063/1.2405420

Oligomer-based organic distributed feedback lasers by room-temperature nanoimprint lithography

Appl. Phys. Lett. 83, 2545 (2003); 10.1063/1.1613362

Photoluminescence and electroluminescence of new lanthanide-(methyoxybenzoyl)benzoate complexes J. Appl. Phys. 82, 1841 (1997); 10.1063/1.365987

\section{Instruments for Advanced Science}

Contact Hiden Analytical for further details: w www.HidenAnalytical.com E info@hiden.co.uk CLICK TO VIEW our product catalogue

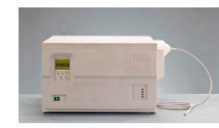

Gas Analysis

dynamic measurement of reaction gas streams catalysis and thermal analysis

molecular beam studies

, fermentation, environmental and ecological studies

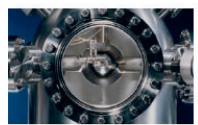

Surface Science UHVTPD

SIMS

end point detection in ion beam etch elemental imaging - surface mapping

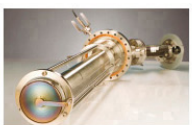

Plasma Diagnostics ; plasma source characterization etch and deposition process reaction kinetic studies , analysis of neutral and radical species

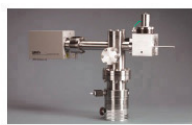

Vacuum Analysis - partial pressure measurement and control of process gases reactive sputter process control , vacuum diagnostics vacuum coating process monitoring 


\title{
Room-temperature nanoimprinting on metallo-organic complexes
}

\author{
Elisa Mele, Dario Pisignano, ${ }^{\text {a) }}$ Marco Mazzeo, Luana Persano, Giuseppe Gigli, \\ and Roberto Cingolani \\ NNL, National Nanotechnology Laboratory of Istituto Nazionale di Fisica della Materia (INFM), \\ c/o Dipartimento di Ingegneria dell'Innovazione, Università di Lecce, via Arnesano, I-73100 Lecce, Italy
}

(Received 26 September 2003; accepted 1 March 2004; published 4 May 2004)

\begin{abstract}
We apply room-temperature nanoimprint lithography to rare-earth metallo-organic complexes, for patterning $600 \mathrm{~nm}$ period gratings onto europium-based $612 \mathrm{~nm}$ light-emitting compounds. After patterning, an increase of up to 6.2 times of the integrated photoluminescence emitted around $\Theta=35^{\circ}$ was observed, resulting from the combination of the effective Bragg scattering induced by the printed grating, with the very narrow-linewidth emission of the metallo-organic complexes. These results are quite promising for the realization of nanoimprinted rare-earth-based optoelectronic devices, such as light-emitting diodes and displays, and distributed feedback elements. (C) 2004 American Vacuum Society. [DOI: 10.1116/1.1714952]
\end{abstract}

\section{INTRODUCTION}

One promising and effective solution to the problem of engineering and controlling the optical properties of lightemitting materials is represented by the use of photonic crystals (PhCs), namely periodic dielectric structures able to favor or to prevent the propagation of light at certain frequencies along certain directions. ${ }^{1,2}$ In particular, the application of PhCs to low-cost organic compounds, allowing excellent luminescence performances and easy synthesis and processing routes, ${ }^{3}$ could be strategic in modern optoelectronics technology. Among different proposed fabrication methods of organic-based PhCs structures, including soft, ${ }^{4}$ electron-beam, ${ }^{5}$ and optical lithography, ${ }^{6,7}$ a cheap and powerful technique is nanoimprinting lithography (NIL), allowing very high throughput and resolution. ${ }^{8}$

The conventional imprinting technology consists of the compression molding of thermoplastic polymers. A mold with an engraved pattern is mechanically pressed onto a substrate coated with a polymeric material. Afterward, the system is heated above the glass transition temperature of the polymer, above which the viscosity strongly decreases. This allows the polymer to conform with the mold pattern. After pressing and subsequent cooling below the glass transition temperature, the mold is removed, and a negative replica of the initial pattern remains on the target. To date, $10 \mathrm{~nm}$ diameter and $40 \mathrm{~nm}$ period holes have been fabricated in polymeric films by NIL. ${ }^{9}$

However, the requirement of the employed molecules to be thermoplastic in nature limits the use of NIL and prevents its use with many low-molar-mass light-emitting molecules. Moreover, when hot embossing is applied to active organic materials, it has to be carried out in a vacuum chamber or in nitrogen atmosphere to avoid the degradation of the optical and electrical properties of the compounds, mainly induced by oxygen incorporation and substitution at high temperature. ${ }^{10}$ Room-temperature nanoimprinting (RT-NIL),

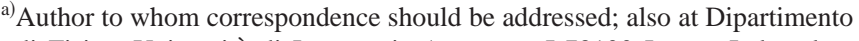
di Fisica, Università di Lecce, via Arnesano, I-73100 Lecce, Italy; electronic mail: elisa.mele@unile.it, dario.pisignano@unile.it initially proposed as alternative manufacturing techniques for patterning thermoplastic polymers, ${ }^{11}$ allows one to fully overcome these drawbacks, as it exploits only an applied pressure to transfer the pattern, thus being suitable for processing of nonthermoplastic compounds under an uncontrolled atmosphere.

In this work, we apply RT-NIL to metallo-organic complexes, patterning films of europium (Eu)-based compounds with periodic $300 \mathrm{~nm}$ features, suitable for the realization of $\mathrm{PhCs}$ and distributed feedback optical devices. ${ }^{12}$ Among rare-earth complexes, which are characterized by high photoluminescent (PL) efficiency, $\eta_{\mathrm{PL}}$, and sharp emission bands, ${ }^{3,13}(\mathrm{Eu})$-based complexes appear very attractive due to their red emission, in view of their wide potential applications in full-color displays and electrically pumped lasers. ${ }^{13-15}$ After patterning, we observed well-defined features, conforming the geometry of the mold. More important, an increase up to 6.2 times of the luminescence emitted around $\Theta=35^{\circ}$ was observed, resulting from the combination of effective Bragg scattering and the very narrowlinewidth emission of the metallo-organic complexes. These results can be very useful for improving the performances of optoelectronic devices based on rare-earth emitting compounds.

\section{EXPERIMENT}

Our lithographic process is schematized in Fig. 1(a). The Eu-based compounds, namely the low-molar-mass molecules tris(benzoylacetonato)-mono(phenanthroline)europium(III) (No. 1) and tris(dibenzoylmethane)-mono(phenanthroline)europium(III) (No. 2) [molecular structures are displayed in Figs. 2(a) and 2(b), respectively] were purchased from H. W. Sands Corp. (FL) and used as received. $3.6 \times 10^{-2} \mathrm{M}$ chloroform solutions were spincoated at 1500 rpm onto Corning glass substrates, thus achieving uniform $300 \mathrm{~nm}$ thick films. Master patterns with parallel grooves of period of $600 \mathrm{~nm}$ and duty-cycle 50\% were first fabricated on silicon by electron-beam lithography by a Raith (Germany) Elphy Plus pattern generator controlling a Leica (Ger- 
(a)
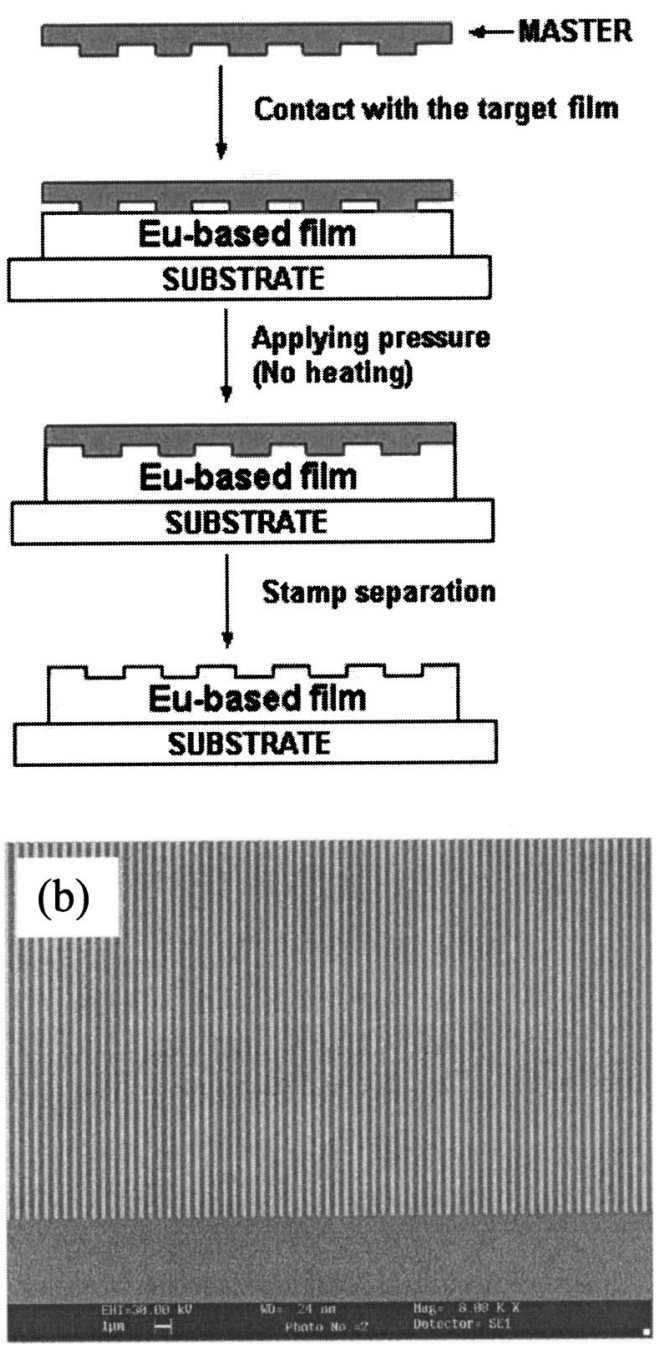

FIG. 1. (a) Schematic diagram of the RT-NIL process (features not in scale). (b) SEM two-dimensional view of the Si master pattern.

many) Stereoscan 440 scanning electron microscope (SEM), and subsequent reactive ion etching by a $\mathrm{CF}_{4} / \mathrm{Ar}$ mixture chosen to minimize the plasma-induced polymer deposition $\left(\mathrm{CF}_{4}-\mathrm{Ar} 86 \%-14 \%\right.$ at $50 \mathrm{sccm}$ with $\mathrm{rf}$ power $=145 \mathrm{~W}$, dc bias $=520 \mathrm{~V}$, and pressure $=70 \mathrm{mTorr}$ ). The obtained stamp, imaged by SEM, is displayed in Fig. 1(b). The masters were then directly employed as a stamp for RT-NIL by a twocolumn precision manual press (PW100 P/O/Weber, Germany), in air, with an applied force of about $4 \mathrm{kN}$. The press consists of two hardened and chromium-plated columns and a top bar, integrating a spindle with a movable upper thrust transmitting piece. The lower pressing plate is mounted on the piston of the hydraulic cylinder. ${ }^{16}$ During the nanoimprinting, the stamp is manually positioned onto a substrate, previously coated with the organic film, and the system (stamp and substrate) is placed onto the lower plate of the precision press. The pressure onto the system is applied by the higher plate of the press, without heating. After pressing, the mold is removed. The overall pressure applied onto the glass substrates (about $1 \times 1 \mathrm{~cm}^{2}$ in size) was around $400 \mathrm{~atm}$
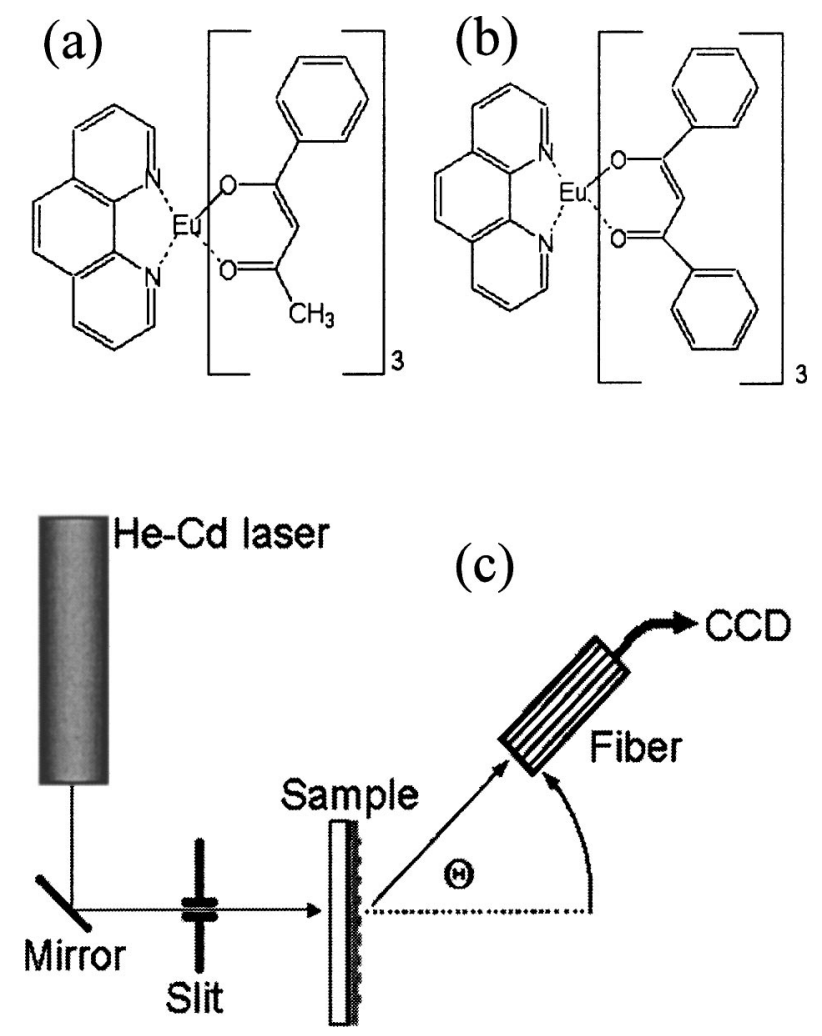

FIG. 2. Molecular structures of the employed rare-earth complexes, No. 1 (a), and No. 2 (b). (c) Schematic diagram of the experimental setup employed for the angle-resolved PL measurements (elements not in scale).

(40 MPa). No antisticking layer was needed in the imprinting process. The PL spectra of the compounds were determined by a fiber-coupled charge coupled device (Ocean Optics, FL) and by exciting with a $\mathrm{He}-\mathrm{Cd}$ laser $(\lambda=325 \mathrm{~nm})$. Angleresolved PL measurements were carried out by collecting the light emitted in a small solid angle ( $\left.\cong 10^{-3} \mathrm{rad}\right)$ around each value of $\Theta$, calculated by moving perpendicularly to the surface grating [experimental setup schematized in Fig. 2(c)].

\section{RESULTS AND DISCUSSION}

Different from conventional (high-temperature) NIL, in RT-NIL the mold is pressed onto the organic film for the time necessary to the pattern transfer, without a wide change of temperature. For this reason, it is particularly suitable for light-emitting molecules, with no need of vacuum or nitrogen environments, which have been previously required to prevent luminescence quenching in light-emitting molecules under high-temperature lithography processes. ${ }^{17}$ Moreover, unlike conventional embossing, in RT-NIL, it is possible to place the master below the sample. In fact, the use of transparent glass substrates facilitates the positioning of the patterned area of the master onto the desired region of the sample, by eye. However, we checked that patterns achieved by either the conventional (master on top) or the inverted (sample on top) imprinting geometry are comparable [inspection by optical and atomic force microscopy (AFM)]. The applied pressure is the key parameter in defining the printing quality and avoiding the detachment of the organic 

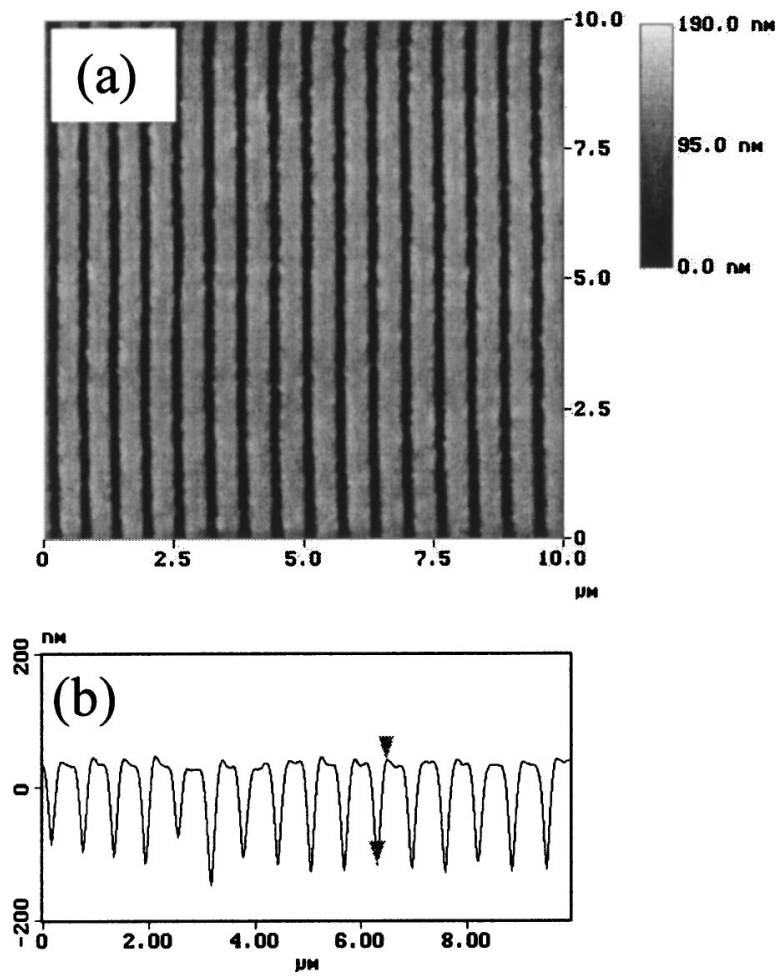

FIG. 3. AFM two-dimensional view of the pattern transfer into compound No. 1 (a) and the corresponding cross section (b).

film from the substrate. Molecules of low-molecular weight generally need low pressure to be imprinted. We adopted pressures comprised between 100 and 600 atm, finding that an incomplete pattern transfer occurs with pressure values below $300 \mathrm{~atm}$, and that pressures higher than $500 \mathrm{~atm}$ determine a loss of adhesion between the organic material and the glass substrate. The process duration was varied between $30 \mathrm{~min}$ and $8 \mathrm{~h}$. An imprinting time of $2 \mathrm{~h}$ was chosen to achieve a complete pattern transfer with an applied pressure of $400 \mathrm{~atm}$.

The plan view and the cross section of a $600 \mathrm{~nm}$ period Eu-based grating (compound No. 1), imaged by AFM, is displayed in Fig. 3, showing a well-defined pattern faithfully reproducing the periodicity of the master. The depth of the imprinted grooves is $(170 \pm 4) \mathrm{nm}$. The achieved resolution depends on the starting master, which suggests the possibility of fabricating $100 \mathrm{~nm}$ features onto rare-earth compounds. Identical results were obtained patterning compound No. 2.

In order to assess the influence of patterning on the emission properties of the metallo-organic complexes, we investigated the PL from both patterned and unpatterned samples as a function of the collection angle, $\Theta$. The emission from compound No. $1\left(\eta_{\mathrm{PL}}=12 \%\right)$ at $\Theta=35^{\circ}$ is shown in Fig. 4 (solid line). The narrow PL from rare-earth complexes origins from the excitation of the $S_{1}$ singlet state of the surrounding organic ligands, whose energy is first transferred to the ligand triplet state, $T_{1}$, via intersystem crossing, and then to the central metal ion. ${ }^{18,19}$ In particular, the emission from Eu-based complexes is peaked at $612 \mathrm{~nm}$, and involves electrons associated with inner $f$ orbital of the central rare-earth

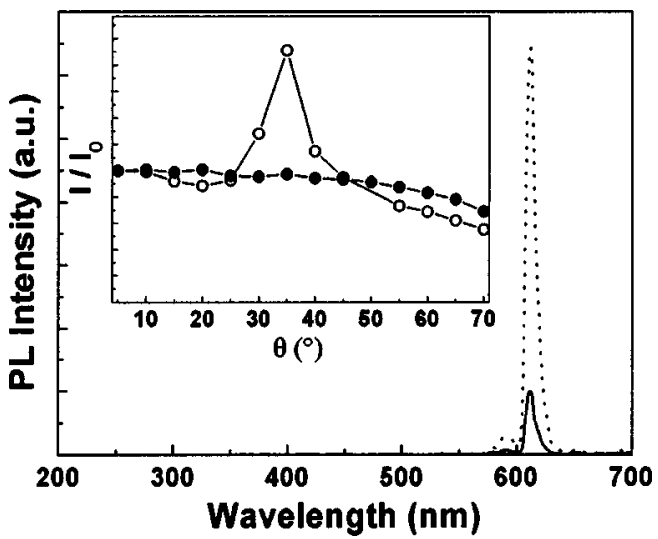

FIG. 4. PL spectra at an emission polar angle, $\Theta=35^{\circ}$, for a patterned (dotted line) and an unpatterned (solid line) Eu-based film. Inset: Angular dependence of the integrated PL emission for a patterned (empty circles) and unpatterned sample (solid circles).

metal ions and correspond to the ${ }^{5} D_{0} \rightarrow{ }^{7} F_{2}$ transition of $\mathrm{Eu}^{3+} \cdot{ }^{13}$ It has been exploited for realizing highly efficient and bright light-emitting devices, ${ }^{18,20}$ reaching an external quantum efficiency as high as $2.1 \%$ and a luminance of 1670 $\mathrm{cd} / \mathrm{m}^{2}{ }^{18}$

Interestingly, the integrated emission from our Eu-based patterned gratings at $\Theta=35^{\circ}$ is more than six times higher than that of the untextured sample (dotted line in Fig. 4). The occurrence of a strong output mechanism from the grating around this emission angle is confirmed by the angular distribution of the integrated PL intensity, I, normalized to the normal intensity, $I_{0}$ (inset of Fig. 4). As opposed to the unpatterned sample, the ratio $I / I_{0}$ of the corrugated films exhibits a remarkable increment for $\Theta$ comprised between $25^{\circ}$ and $45^{\circ}$, being maximum at $\Theta=35^{\circ}$. Compound No. 2 exhibits a similar behavior.

The insertion of a one-dimensional wavelength-scale periodic nanostructure into light-emitting films strongly enhances the forward output from the active compound, because photons are preferentially scattered along certain directions, instead of being guided along the slab. 6,7 The imposed grating enables the light trapped in waveguide modes to be Bragg scattered out of the film along the forward direction, reducing the self-absorption within the organic slab. The emission angle of the output wavelength, $\lambda$, is related to the cavity geometry by the relation:

$$
\frac{2 \pi}{\lambda} \sin \Theta= \pm \frac{2 \dot{\pi} n_{\mathrm{eff}}}{\lambda} \pm m \frac{2 \pi}{\Lambda}
$$

where $n_{\mathrm{eff}}$ is the effective refractive index of the waveguided mode, $\Lambda$ is the grating period, and $m$ is an integer. The scattering process is therefore governed by the coupling condition of the guided photons to the free radiation, namely by the conservation of the in-plane component of the wave vector of the output light, $k_{0}= \pm 2 \pi / \lambda$, and of the guided mode, $k_{g}= \pm 2 \pi n_{\text {eff }} / \lambda$, by the Bragg vector (PhC reciprocal lattice vector), $G=2 \pi / \Lambda$. By means of this radiation coupling to free radiation, the use of planar $\mathrm{PhCs}$ within both inorganic ${ }^{21}$ and organic ${ }^{6,7}$ light-emitting diodes can also be exploited to 
increase the device forward luminance. According to Eq. (1), the guided radiation within our Eu-based organic slab experiences an effective refractive index of about 1.6.

For each value of $\Theta$, the Bragg scattering from patterned gratings generally gives rise to one or two evident peaks, superimposed to the broad luminescence spectra of lightemitting organic compounds. ${ }^{7}$ For our printed gratings, these extra peaks have a linewidth up to $50 \mathrm{~nm}$, as we could establish by previous RT-NIL experiments with the same masters, onto organic dyes compounds with broad emission. In the case of the Eu-based complexes, due to their very narrow emission band, the printed corrugation results therefore in an enhancement of the whole PL spectrum for $\Theta$ around $35^{\circ}$. This can be very important in employing rare-earth metalloorganic complexes in optoelectronic devices, in controlling the emission directionality, and in enhancing the light output.

\section{CONCLUSION}

We have demonstrated that RT-NIL is suitable for patterning rare-earth-based metallo-organic complexes, by transferring well defined $600 \mathrm{~nm}$ period gratings onto Eu-based light-emitting films. The output light emitted at $\Theta$ around $35^{\circ}$ is enhanced by more than six times with respect to the unpatterned samples, due to the Bragg scattering induced by the printed gratings. These results open the way to the realization of rare-earth-based printed optoelectronic devices, like textured light-emitting diodes and distributed feedback microcavities, and are very promising also in view of the possibility of employing rare-earth complexes emitting in the infrared, such as $\mathrm{Nd}^{3+}(\lambda=1.34 \mu \mathrm{m})$ - and $\mathrm{Er}^{3+}$ $(\lambda=1.53 \mu \mathrm{m})$-based compounds, for application in telecommunications.
${ }^{1}$ J. D. Joannopoulos, R. D. Meade, and J. N. Winn, Photonic CrystalsMolding the Flow of Light (Princeton University Press, Princeton, NJ, 1995).

${ }^{2}$ E. Yablonovitch, Phys. Rev. Lett. 58, 2059 (1987).

${ }^{3}$ A. Kohler, J. S. Wilson, and R. H. Friend, Adv. Mater. (Weinheim, Ger.) 14, 701 (2002).

${ }^{4}$ J. R. Lawrence, P. Andrew, W. L. Barnes, M. Buck, G. A. Turnbull, and I. D. W. Samuel, Appl. Phys. Lett. 81, 1955 (2002).

${ }^{5}$ C. Liguda, G. Böttger, A. Kuligk, R. Blum, M. Eich, H. Roth, J. Kunert, W. Morgenroth, H. Elsner, and H. G. Meyer, Appl. Phys. Lett. 78, 2434 (2001).

${ }^{6}$ J. M. Lupton, B. J. Matterson, I. D. W. Samuel, M. J. Jory, and W. L. Barnes, Appl. Phys. Lett. 77, 3340 (2000).

${ }^{7}$ B. J. Matterson, J. M. Lupton, A. F. Safonov, M. G. Salt, W. L. Barnes, and I. D. W. Samuel, Adv. Mater. (Weinheim, Ger.) 13, 123 (2001).

${ }^{8}$ S. Y. Chou, P. R. Krauss, and P. J. Renstrom, Appl. Phys. Lett. 67, 3114 (1995); Science 272, 85 (1996).

${ }^{9}$ S. Y. Chou, P. R. Krauss, W. Zhang, L. Guo, and L. Zhuang, J. Vac. Sci. Technol. B 15, 2897 (1997).

${ }^{10}$ M. Yan, L. J. Rothberg, F. Papadimitrakopoulos, M. E. Galvin, and T. M. Miller, Phys. Rev. Lett. 73, 744 (1994).

${ }^{11}$ D.-Y. Khang, H. Yoon, and H. H. Lee, Adv. Mater. (Weinheim, Ger.) 13, 749 (2001).

${ }^{12}$ H. Kogelnik and C. V. Shank, Appl. Phys. Lett. 18, 152 (1971).

${ }^{13}$ Z. Hong, C. Liang, R. Li, W. Li, D. Zhao, D. Fan, D. Wang, B. Chu, F. Zang, L.-S. Hong, and S.-T. Lee, Adv. Mater. (Weinheim, Ger.) 13, 1241 (2001).

${ }^{14}$ Y. Kawamura, Y. Wada, Y. Hasegawa, M. Iwamuro, T. Kitamura, and A. Yanagida, Appl. Phys. Lett. 74, 3245 (1999).

${ }^{15}$ R. J. Curry and W. P. Gillin, Appl. Phys. Lett. 75, 1380 (1999).

${ }^{16} \mathrm{http}: / / \mathrm{p}$-o-weber.de/e/presses/pw_e.html

${ }^{17}$ J. Wang, X. Sun, L. Chen, and S. Y. Chou, Appl. Phys. Lett. 75, 2767 (1999).

${ }^{18}$ P. Sun, J. Duan, H. Shih, and C. Cheng, Appl. Phys. Lett. 81, 792 (2002).

${ }^{19}$ J. Kido, H. Hayase, K. Hongawa, and K. Nagai, Appl. Phys. Lett. 65, 2124 (1994).

${ }^{20}$ C. Adachi, M. A. Baldo, and S. R. Forrest, J. Appl. Phys. 87, 8049 (2000).

${ }^{21}$ A. A. Erchak, D. J. Ripin, S. Fan, P. Rakich, J. D. Joannopoulos, E. P. Ippen, G. S. Petrich, and L. A. Kolodziejski, Appl. Phys. Lett. 78, 563 (2001). 